\title{
Microleakage and Resin-to-Dentin Interface Morphology of Pre-Etching versus Self-Etching Adhesive Systems
}

\author{
G.L. Waldman, T.K. Vaidyanathan* and J. Vaidyanathan
}

NJ Dental School, University of Medicine and Dentistry of New Jersey, Newark, NJ 07103, USA

\begin{abstract}
The purpose of this study was to compare the microleakage and tissue-adhesive interface morphology from Class V restorations using different systems of dentin adhesives. Class V cavities were prepared on buccal surfaces of 27 extracted caries-free molars and premolars. Teeth were randomly assigned to one of three groups: (1) Prime \& Bond NT, a $5^{\text {th }}$ generation system using an initial step of total etch followed by a second step of application of a self bonding primer (2) Clearfil SE Bond, a $5^{\text {th }}$ generation adhesive system employing two separate steps of self-etch priming and subsequent bonding (3) One-up Bond F, a $6^{\text {th }}$ generation one step self-etching, self-priming and self-bonding adhesive. Microleakage and interface morphology of teeth restored with these adhesives and a composite resin were evaluated. Kruskal-Wallis Test $(\mathrm{p}=0.05)$ was used to analyze the results. SEM analysis was used to relate interface morphology to microleakage. The mean and (SD) values of microleakage were: Prime and Bond NT: 0.15 (0.33), Clearfil SE Bond: $0.06(0.17)$ and One-up Bond F: 2.96 (0.63). The mean microleakage for One-up Bond was significantly higher than for the other groups ( $\mathrm{p}<0.05)$. Protruding tags in dentin channels were observed in Prime and Bond and Clearfil systems, but not in One-up Bond. The single step adhesive system, although more convenient for the clinician, uses a low viscosity formulation difficult to keep in place on cavity walls. It also tends to be too aggressive and hydrophilic to create an impermeable hybridized tissue-adhesive interfacial layer resistant to microleakage. Two-step adhesive systems, on the other hand, were retained on all segments of the cavosurface during application, and formed a hybridized interfacial layer resistant to microleakage.
\end{abstract}

\section{INTRODUCTION}

The conventional acid-etch technique pioneered by Bounocore [1] for composite resin restorations has been used in dentistry for almost 50 years. One of the major threats to the long-term success of resin restorations is microleakage. Microleakage may be defined as the passage of bacteria, fluids, molecules, or ions between the cavity wall and the restorative material applied to it [2]. Microleakage is associated with a number of clinical conditions including, sensitivity, recurrent caries, staining of the restoration margins, pulpal damage, and breakdown of the restorative material. Therefore, prevention of microleakage is an important consideration when developing an adhesive system for dental restorative applications.

The majority of currently popular adhesive systems were developed under total etch technique during the last two decades. They were designed with three separate steps in the bonding process: etching, priming and bonding, and such systems have been designated as " 4 th" generation adhesives [3]. Over the years, advances in adhesive dentistry have led to the development of other adhesives to facilitate greater bond strength, sealing of tissue permeability, and above all, convenience and ease of application. Some popular adhesives used today are the "5th generation" systems. Two such

*Address correspondence to this author at the Dept. of Restorative Dentistry, NJ Dental School, UMDNJ, Newark, NJ 07103, USA; Tel: (973) 972-6250; Fax: (973) 972-0370; E-mails: vaidyatk@umdnj.edu and vaidyatk@hotmail.com systems are in use. In one type, the primer and adhesive are combined in a single bottle, but a pre-bonding step of total acid etching is still required [3]. These systems have been shown to be effective in preventing microleakage in several studies [4]. During the past decade, self-etching primer systems have also been introduced under these " 5 th generation" formulations. These self-etching primers alleviate the need for a separate step of acid etching and eliminate rinsing and drying steps, but require a separate application of an adhesive resin or bonding agent. More recently, "6th generation" adhesive systems have been introduced. These systems greatly simplify the adhesive process even further by incorporating the etchant, primer, and bonding agent into a single formulation for a one-step application. Such formulations can also be designed to incorporate materials to release fluoride for therapeutic benefits and color change markers to indicate completion of polymerization [5].

While many studies have investigated the bond strength and marginal adaptation of self-etching primers [6-12], other studies have also compared the microleakage and interface morphology at the margins of restorations placed using these new materials along with some total etch formulations [825]. Some of these studies have demonstrated that selfetching primers and the conventional etch, prime and bonding adhesive systems are equally effective in preventing microleakage [8-13]. However, other studies have reported that separate etching, priming and bonding steps are more effective in reducing microleakage [15-21]. There is therefore a need to examine the microleakage of specific adhesive 
systems with significant formulation or technique differences. The main purpose of the present study was to compare the microleakage in restored teeth using a single step adhesive system (one-up Bond F) with two-step systems (Clearfil SE Bond, Prime and Bond NT). The second objective of the study was to examine the morphology of the interfacial region in each system to explore its effect on potential microleakage differences. The null hypotheses tested in this investigation were: (1) Microleakage from Class V cavities using single step and two-step adhesive systems evaluated are not significantly different. (2) The tissue-adhesive interface is not significantly influenced by the adhesive systems.

\section{MATERIALS AND METHODS}

\section{Experimental Layout and Sample Size Estimate}

The number of teeth to be studied in the microleakage analysis was estimated based on a power analysis using a one-factor, three-level design (three types of adhesives). The power analysis was carried out at a significance level of $\alpha=$ 0.05 with assumed values of expected variation in microleakage $(\sigma)$ and minimum detectable difference in measured values of microleakage $(\delta)$, based on previously reported research results [22]. The total number of teeth for all study groups was selected as $\mathrm{N}=27$ corresponding to $>90 \%$ power.

Thirty-three recently extracted (up to 3 months) cariesfree human molars and premolars were included. After hand scaling, teeth were stored in $0.1 \%$ Thymol at $4{ }^{\circ} \mathrm{C}$. Twentyseven teeth (18 molars and 9 premolars) were used for microleakage evaluation and the remaining 6 teeth were saved for SEM analysis.

\section{Microleakage Evaluation}

Class V cavities were prepared in the middle third of the buccal surface of each tooth. The dimensions of each preparation were approximately $3 \mathrm{~mm}$ wide x $2.5 \mathrm{~mm}$ deep x $2 \mathrm{~mm}$ long occlusal-cervically. One operator prepared all teeth with standard \#330 burs (SS White FG, type 2, Class 4A, SS white Co, Lakewood, NJ) in a high speed, water-cooled handpiece. Burs were replaced after every five preparations. Prepared teeth were randomly assigned to one of three adhesive groups, with 9 teeth per group ( 3 premolars and 6 molars). The three groups were restored using different adhesive systems as follows:(1) Total etch + Prime \& Bond NT (Dentsply/Caulk, Milford, DE, USA) application, (2) Clearfil SE Bond (Kuraray Medical Inc., Kurashiki, Okayama, Japan) application + Bonding (3) One-up Bond F (Tokuyama Dental Corp., Taitou-ku. Tokyo, Japan) application. These systems are designated as PNB, SEB and OUP, respectively, in this study. The adhesive systems were placed according to manufacturer's instructions and then restored with a hybrid composite (TPH, Dentsply/Caulk). Teeth were stored in deionized water at $37^{\circ} \mathrm{C}$ in a humidity chamber (maintained at $95 \%$ relative humidity) for 24 hours prior to thermocycling for 2 days (approximately 1000 cycles). Thermocycling was achieved with a programmed robot (Microbot model TeachMover, Questtech, MI.) that alternated samples between two temperature controlled water baths at $4{ }^{\circ} \mathrm{C}$ and $60^{\circ} \mathrm{C}$, respectively with a dwell time of $60 \mathrm{sec}$ in each bath. After thermal cycling, the entire surface of each tooth (with the exception of the restoration and $1 \mathrm{~mm}$ of tooth structure adjacent to the restoration) was covered with two coats of nail varnish (NailSlicks, CG) to prevent dye penetration into the tooth except at the resin-to-tooth interface. Samples were placed in $2 \%$ basic fuchsin dye solution $(\mathrm{pH}=5.8)$ for 24 hours at $37^{\circ} \mathrm{C}$ and $95 \%$ humidity. Samples were sectioned with an Isomet slow-speed saw (Beuhler Ltd, Evanston, IL) with a diamond blade in water, longitudinally in a buccolingual direction yielding a $1-1.5 \mathrm{~mm}$ section through the center of the restoration. Dye penetration was measured using a dissecting microscope at $2.5 \mathrm{x}$ magnification according to the following 5 point interval scale [15]: $0=$ no leakage, 1 $=$ leakage restricted to the enamel, $2=$ leakage into dentin but not reaching the axial cavity wall, 3 = leakage reaching the axial cavity wall, 4 = leakage beyond the axial cavity wall reaching the pulp (see Fig. 1). All evaluations were carried out in a blind study with no information to the examiner about the identity of the adhesive system used. One examiner measured each section separately resulting in 54 data points (27 teeth $\mathrm{x} 2$ sections). Dye penetration was re-measured one month later by the same examiner and a second independent examiner to determine within-rater and between-rater reliabilities, respectively. A consensus measurement was forced for all disagreements, if any.

Results were analyzed statistically by (a) bivariate fit to determine intra- and inter-rater evaluation reliability, (b) plots of microleakage least square (LS) means against tooth types (molar vs. premolars) to determine any variability associated with the tooth type, (c) quantile box plots to characterize the distribution and central trends of microleakage data, and (d) Kruskal-Wallis test to determine significant microleakage differences between adhesives of rank sums in microleakage scores. All statistical analysis procedures were performed on JMP statistical software (SAS Institute, Cary, N.C.).

\section{SEM Analysis}

Dentin discs with a thickness of $1.0-1.5 \mathrm{~mm}$ were prepared from six caries-free human molars. The occlusal third of each tooth was removed using the Isomet slow-speed saw to expose the dentin surface. A second parallel cut to the previous cut was used to obtain a dentin disc. Wet sanding using 600-grit silicon carbide for 1 minute in a manual metallographic polishing set up (Handimet 2, Buehler, Lake Bluff, IL) produced standardized smear layers [18]. The discs were washed in water, plunged into ethanol, and dried with moisture-free air [19]. The discs were randomly assigned to one of three adhesive groups described in the microleakage procedure ( $\mathrm{n}=2$ /group). The adhesives were applied according to according to manufacturer's instructions followed by the application of a $1 \mathrm{~mm}$ layer of hybrid composite (TPH) and light cured for 40 seconds. The resinbonded discs were cross-sectioned perpendicular to expose the resin-dentin interface, followed by polishing to a high gloss with 600-grit emery paper and $0.05 \mu \mathrm{m}$ alumina under a stream of water. The sections were demineralized in hydrochloric acid $(\mathrm{HCl})$ solution $(6 \mathrm{~mol} / \mathrm{L})$ for $10 \mathrm{sec}$, and deproteinated in $1 \%$ sodium hypochlorite $(\mathrm{NaOCl})$ solution for 10 min., sputter-coated with gold and examined in a Hitachi SEM model S-2500 using an accelerating voltage of $15.0 \mathrm{kV}$ [26]. The micromorphology at the dentin-restoration interface was visualized under SEM for each adhesive system. 

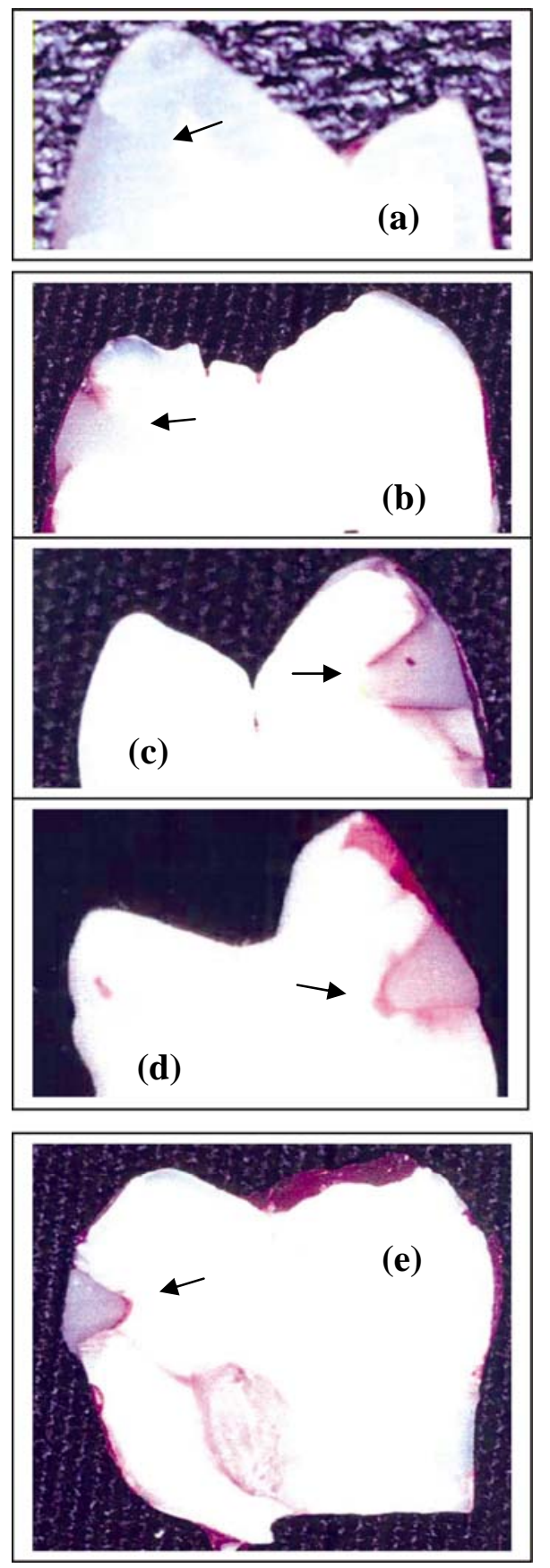

Fig. (1). Illustration of the microleakage interval scale. (a) no leakage $=0$, (b) leakage restricted to the enamel $=1$, (c) leakage into dentin but not reaching the axial cavity wall $=2$, (d) leakage reaching the axial cavity wall $=3$, (e) leakage beyond the axial cavity wall reaching the pulp $=4$. The restoration locations marked with arrows.

\section{RESULTS}

\section{Microleakage}

The mean and (SD) of microleakage scores varied from a low of $0.06(0.17)$ for self-etching system SEB and 0.15 (0.33) for the total etch two-step system PNB to a high of $2.96(0.63)$ for single step system OUP. The median scores (0 for PNB and SEB, 2.67 for OUP) also showed clear dif- ferences. Thus, initial analysis revealed important differences in microleakage occurrence between groups.

The within-rater reliability and between-rater reliability, based on bivariate fitting of interval data (at two time points) to a normal ellipse model $(\mathrm{P}=0.95)$ on the JMP platform, were better than $98.8 \%$ and $99.8 \%$, respectively. This facilitated proper comparisons between different adhesive systems based on the evaluator scores. Comparison of mean microleakage LS means of molars and premolars also showed no significant differences in microleakage with respect to tooth type both cumulatively as well as individually in each group. Consequently, subsequent statistical analysis compared adhesives without regard to tooth type.

Fig. (2) presents quantile box plots of the microleakage scores. The plot illustrates the location of the middle half of the data (from the lower quartile to the upper quartile) as well as the tails of the distribution.

Comparisons of the quantile box plots indicate that the distributions of the microleakage scores of OUP are centered at a high median score of 2.67 compared to PNB and SEB, which are centered at a median score of 0 . Kruskal-Wallis test of ranked microleakage scores and chi-square approximation revealed that OUP demonstrated significantly higher mean microleakage $(p<0.0001)$ than SEB and the PNB systems (Table 1).

However, there was no significant difference in microleakage between the two-step self-etch SEB and the twostep total etch PNB systems ( $\mathrm{p}>0.05)$.

\section{Interfacial Micro-Morphology}

Split SEM photomicrographs of representative specimens for each adhesive at two magnifications of 200x and 1000x are presented in Fig. (3a, b, c). A true hybrid layer exhibiting resin permeation into demineralized dentin, including resin tags protruding into the dentinal tubules, characterized the morphology at the dentin-resin interface for PNB. The SEB system also showed a hybrid layer and protruding plugs to the tubular orifices. In contrast, the interfacial morphology with the OUP adhesive was characterized by a thick hybrid

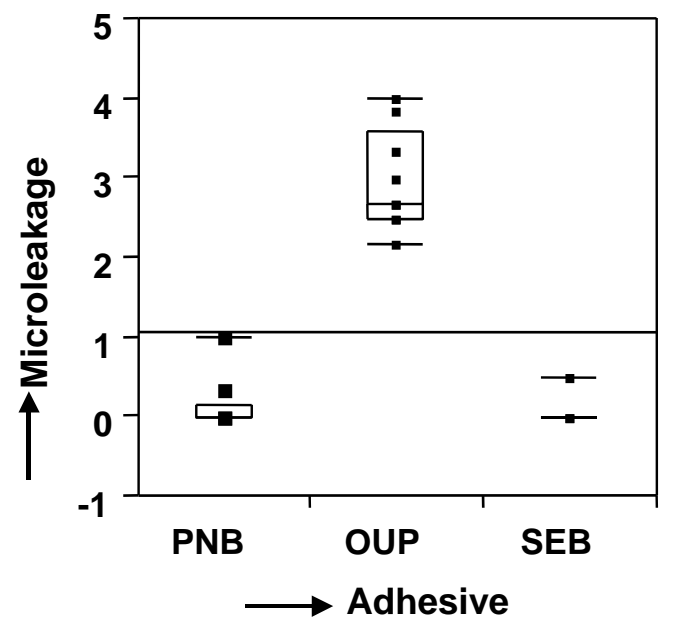

Fig. (2). Quantile box plot and data distribution of microleakage scores. The plot also shows the median scores for different adhesives (0 for SEB and PNB, 2.67 for OUP), indicating the central trends of microleakage data showing important differences. 
layer, but without any clear indication of resin tags sealing the tubular openings.

Table 1. Summary of Kruskal Wallis Test and Chi Square Approximation of $\mathrm{H}$ - Statistic

\begin{tabular}{|c|c|c|c|}
\hline Level & Count & Score Mean & (Mean-Mean0)/Std0 \\
\hline \hline PNB & 9 & 10.0000 & -2.006 \\
\hline OUP & 9 & 23.0000 & 4.549 \\
\hline SEB & 9 & 9.0000 & -2.515 \\
\hline
\end{tabular}

1-way Test, ChiSquare Approximation

\begin{tabular}{|c|c|c|}
\hline ChiSquare & DF & Prob>ChiSq \\
\hline \hline 21.0376 & 2 & $<.0001$ \\
\hline
\end{tabular}

\section{DISCUSSION}

Previous studies have suggested that the ability of a restorative material/adhesive to seal the interface with tooth structure is the most significant factor in determining resistance to secondary caries [25]. These studies have also demonstrated that a hybridized resin-tissue layer with resin tags into tubules and adhesive permeation into demineralized porous sub-surfaces of intact dentin establish the bonding mechanism to seal and prevent the permeability through dentin [24-26].Others have suggested that the formation of hybridized zone involves secondary bonding interactions at the atomic level, which may include van der Waals forces, electrostatic interactions and hydrogen bonding between collagen fibrils and the primer [27]. A layer of interaction zone containing an intimate mixture of resin and components of dentin at the molecular level capable of reducing permeability across the interface is thus formed [26]. For this reason, microleakage and interfacial adhesive forces involving tissues and bonding systems have been examined in many published reports and reviews in the past.

Several studies have examined the bond strength of some of the newer 5th and 6th generation bonding systems. However, the bond strength of an adhesive does not always accurately predict its sealing ability [28]. Thus, adhesives with high bond strength may still exhibit undesirable levels of microleakage. The evaluation of microleakage for each adhesive is however very important.

This investigation was carried out to compare the microleakage of three distinctly different formulations, SEB, PNB, and OUP. The results of this study show that microleakage from Class V cavities using SEB is comparable to the total-etch PNB system. The OUP system demonstrated significantly more microleakage than the other two systems tested. These results are consistent with previous studies that evaluated other self-etching primers and one-step adhesive systems. The self-etching primer system, Clearfil Liner Bond (LB) has been shown in many studies to effectively prevent microleakge [8-29]. Other studies, however, have reported poor sealing ability of self-etching primers [20]. Likewise, another sixth generation adhesive system, Prompt L-pop, demonstrated poor marginal adaptation in previous studies [30]. There is no previous report of any comparative microleakage studies on the different adhesive systems used in this study. However, data reported in a recent limited evaluation by Reality [31] give lower mean microleakage values

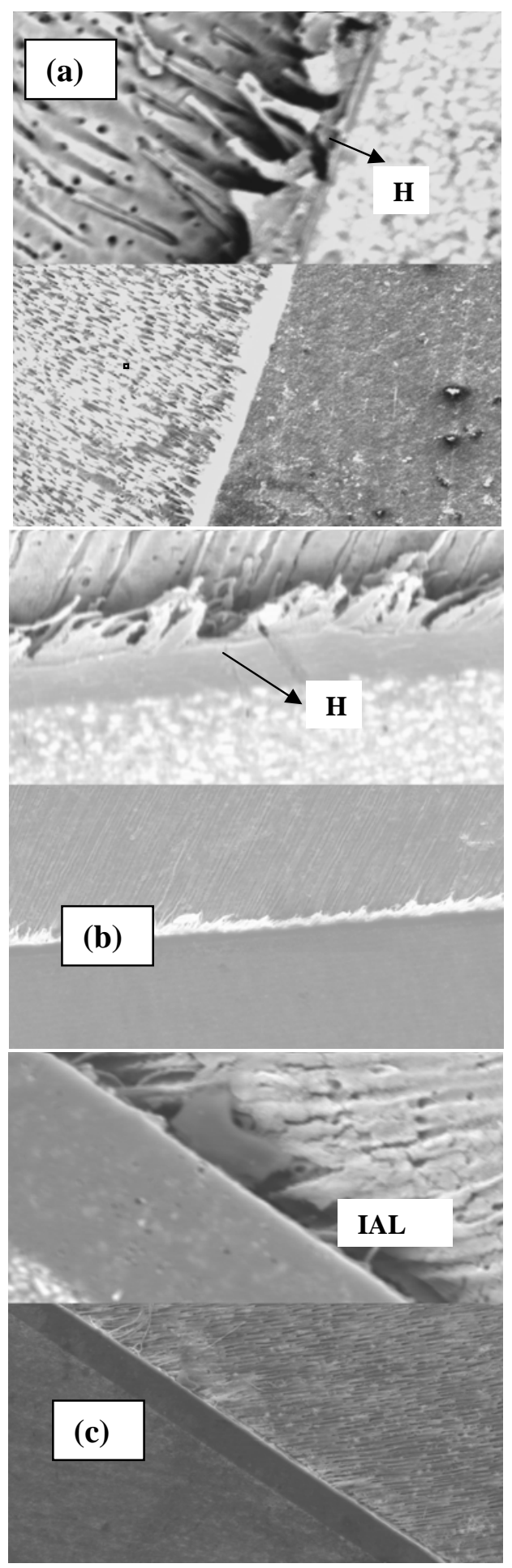

Fig. (3). Split micrographs (200X bottom/1000X top) of dentinadhesive interfacial morphology (a) PNB (b) SEB and (c) OUP. The hybrid layer is marked ' $\mathrm{H}$ ' in all images. Note the distinct hybrid layer morphology in PNB and SEB. In OUP, a thick interfacial adhesive layer (IAL) appears to have fully dissolved the smear layer including smear plugs into the tubules. 
for SEB and PNB, and a higher value for OUP, similar to the trend of mean values for these three adhesives reported in this study.

The SEB system is composed of a self-etching primer with a separate bonding agent. The primary active agents in the self-etching primer and bonding agent are 10methacryloyloxydecyl dihydrogen phosphate (10-MDP) and hydroxyethyl methacrylate (HEMA). The use of 10-MDP as self-etching primer has been reported to cause minimal dissolution of smear plugs at the tubular openings and thus reduces dentin permeability through tubular pathways. In addition, it is able to demineralize and permeate into the subsurfaces of intact dentin below the smear layer. According to Kubo et al, the presence of two hydroxyl groups in the 10MDP structure may facilitate its chelation with the calcium ions of dentin and enamel, and thus help reduce permeability [32]. Yoshida et al. have also shown evidence of chemical bonding between MDP and HAP structure [33]. The primer also contains hydrophilic dimethacrylate to promote wetting while the bonding agent has hydrophobic dimethacrylate and Bis-GMA to promote adhesion to the resin material. Previous studies have shown that these agents demonstrate good adhesion to both dentin and enamel [5]. Thus it is not surprising that this material performed well in this study. The OUP adhesive system uses a mixture of methacrylated phosphoric acid monomer and MAC -10 (a 10-carbon chain monomer with two carboxylic acid ends) to aggressively demineralize dentin. Recently reported data [31] indicate that the dentin-restoration mean bond strength associated with the use of OUP (18.1 MPa) is comparable to that of PNB $(21.7 \mathrm{MPa})$. However, our results show a much greater tendency for microleakage in the former compared to the latter. This would indicate that the relationship between microleakage and bond strength might be complicated. It is however possible to explain this disconnect by an analysis of the differences in the testing conditions for bond strength and microleakage evaluation used in typical testing methods. Bond strength measurements are carried out in planar sections of tooth, where the operator is able to control the adhesive spread more effectively to establish uniformly effective bonding. Microleakage is measured in 3-D cavity configurations. The low viscosity and poor thixotropic behavior associated with the OUP makes the adhesive to flow without operator control after placement. This makes it difficult to retain the adhesive at some cavity walls where gravity effects help the flow away from the surface. In contrast, the higher viscosity and thixotropic behavior of the PNB and SEB allow them to remain at the placement location until spread uniformly under application pressure, and thus help penetrate into the substrate surfaces in all 3-D cavity segments. Thus the inability to retain the adhesives of lower viscosity at all cavity segments may be an important factor leading to the observed higher microleakage in some adhesive systems. Improved thixotropic properties of the adhesive formulation is necessary to retain the adhesive when placed on the cavity wall and to allow it to flow under shear stress These results are consistent with previous studies, which suggested that the new sixth generation systems cannot be kept in place, and must be refreshed continuously [34].

In this study, the resin-dentin interfacial morphology was observed for each adhesive.The SEM photomicrographs show significant differences in the interfacial morphology of the three adhesive systems. The total etch systems are believed to create a micromechanical interlocking adhesion mechanism due to hybridization of demineralized tissue with adhesive primer [35]. The interactions at the dentin interface are especially important because of the critical role of the resin-dentin hybrid layer in bonding and sealing at this interface. This morphology can be clearly visualized by SEM analysis, and many authors have identified the hybridized layer by this method. Our results confirm the presence of the hybrid layer and the resin plugs at the tubular openings at the interface in the PNB system. A hybrid layer and plugs at the tubular openings also characterized SEB interface with dentin. It is believed that these plugs are smear plugs formed during the cavity preparation, which may have been modified by interaction with the primer. The interaction of SEB with smear layer and the smear plugs is considered to be relatively mild [36], and may allow the original smear plugs to remain in a modified form, rather than be completely dissolved by the primer. However, it has been pointed out in previous reports that the aggressiveness of the self-etching primer may change the morphology of the hybrid layer at the tissue-resin interface [36]. When the $\mathrm{pH}$ is very low (as is the case with OUP system), the reactant will completely dissolve the smear layer including the smear plugs at the tubular orifices. This tends to produce a thick hybrid layer, but may fail to seal the tubules effectively causing potential for increased microleakage. Our results indicate an interfacial morphology in OUP system where the smear layer and the resin plugs at the tubular orifices may be dissolved into the adhesive. This can potentially cause shrinkage effects, which may generate discontinuities at the dentin-adhesive interface. During thermal cycling, the additional thermal stresses generated may cause separation at the interface creating pathways for microleakage. It has also been reported that excessive hydrophilic nature of OUP may also lead to "osmotic blistering" causing water filled channels or "water trees" within the adhesive, causing increased permeability in this system [37].

From the results of this study it is concluded that the fifth generation adhesives demonstrated superior margin sealing than the sixth generation adhesive. The fifth generation adhesives show low micro-leakage values similar to reported results of fourth generation adhesives, which use etching, priming and bonding steps. Furthermore, many practitioners have reported a reduction in post-operative sensitivity with the use of self-etching primers when compared to the totaletch technique [26-38]. Self-etching agents do not remove the smear layer and the smear plugs, but only modify these structures, consequently the tubular orifices remain sealed by the plugs. It is believed that this mechanism of adhesion, that enables bonding without exposing the dentinal tubules, is responsible for eliminating post-operative sensitivity. Thus, self-etching primers may have the advantage of reducing sensitivity in addition to a reduced microleakage and ease of application. In contrast, the one-step adhesive system demonstrated significantly higher level of microleakage in this study, although its application is designed to be simpler.

\section{CONCLUSIONS}

Although it is desirable from a clinician's point of view to reduce the number of steps involved in adhesive bonding, the adverse effects of reducing individual steps need to be carefully considered. One-step bonding formulations tend to 
use more chemically aggressive, excessively hydrophilic and low viscosity formulations for simultaneous etching, priming and bonding effects, but such adhesives tend to be difficult to keep in place and are far too aggressive and hydrophilic to create an efficient tissue-adhesive hybridized layer to ensure adequate sealing at the dentin-restoration interface. Fifth generation bonding systems, which use two steps (of either etch and bond with self priming bonding agent or prime with a self-etching primer and bond) are good alternatives to totaletch three-step (etch, prime and bond) adhesive systems, but single step OUP needs additional optimization in order to control microleakage. The low viscosity, low $\mathrm{PH}$ and high hydrophilicity of this adhesive may adversely affect its ability to effectively seal the dentin-adhesive interface.

\section{ACKNOWLEDGEMENTS}

Partial support for this research from NIH-NIDCR grant \#R01DE14370 is gratefully acknowledged. This research was carried out by GLW as part of her research requirement for Advanced certification in Pediatric Dentistry

\section{REFERENCES}

[1] Bounocore MG. A simple method of increasing the adhesion of acrylic filling materials to enamel surfaces. J Dent Res 1955; 34 : 849-53.

[2] Jensen ME, Chan DC. Polymerization shrinkage and microleakage. In: Vanherle G, Smith DC, Eds. International symposium on posterior resin dental restorative materials. Ultrect, The Netherlands: Peter Szulc Publishing Co 2003, pp. 243-62.

[3] Kugel G, Ferrari M. The science of Bonding: From first to sixth generation. J Am Dent Assoc 2000; 131(Suppl): 20-5.

[4] Al-Ehaideb A, Hohammed H. Microleakage of "One Bottle" dentin adhesives. Oper Dent 2001; 26: 172-5.

[5] Product brochure for One-Up Bond F System. J Morita USA Inc, Calfornia USA.

[6] Chigira H, Yukitani W, Hasegawa T, et al. Self-etching dentinal primers containing Phenyl-P. J Dent Res 1994; 73: 1088- 95.

[7] Watanabe I, Nakabayashi N, Pashley DH. Bonding to ground dentin by a Phenyl-P self-etching Primer. J Dent Res 1994; 73: 121220 .

[8] Abdalla AI, Davidson CL. Shear bond strength and microleakage of new dentin bonding systems. Am J Dent 1993; 6: 295-8.

[9] Sano H, Yoshikawa T, Pereira PNR, et al. Longterm durability of dentin bonds made with a self-etching primer, in vivo. J Dent Res 1999; 78: 906-11.

[10] Burrow MF, Satoh M, Tagami J. Dentin bond durability after three years using a dentin-bonding agent with and without priming. Dent Mater 1996; 12: 302-7.

[11] Hayakawa T, Kikutake K, Nemoto K. Influence of self-etching primer treatment on the adhesion of resin composite to polished dentin and enamel. Dent Mater 1998; 14: 99-105.

[12] Nakabayashi N, Sami Y. Bonding to intact dentin. J Dent Res 1996; 75: 1706-15.

[13] Yap AUJ, Ho KS, Wong KM. Comparison of marginal sealing ability of new generation bonding systems. J Oral Rehabil 1998; 25: 666-71.

[14] Tulunoglu Ö, Üctash M, Alacam, Ömürlü H. Microleakage of light-cured resin and resin-modified glass-ionomer dentin bonding agents applied with co-cure vs pre-cure technique. Oper Dent 2000; 25: $292-8$.
[15] Gagliardi RM, Avelar RP. Evaluation of microleakage using different dentin bonding agents. Oper Dent 2002; 27: 582-6

[16] Ahasham SSA. An in vitro study of microleakage around class V cavities bonded with self-etching versus a conventional two-bottle system. Prim Dent Care 2006; 13: 107-11.

[17] Guéders AM, Charpentier JF, Albert AI, Geerts SO. Microleakage after thermocycling of 4 etch and rinse and 3 self etch adhesives with and without a flowable composite lining. Oper Dent 2006; 31 : 450-5.

[18] Silveira de Araújo C, Incerti da Silva T, Oqliari FA, Meireless SS, Piva E, Demarco FF. Microleakage of seven adhesive systems in enamel and dentin. J Contemp Dent Pract 2006; 31: 26-33.

[19] Owens BM, Johnson WW, Harris EF. Marginal permeability of self-etch and total etch adhesive systems. Oper Dent 2006; 31: 60-7

[20] Opdam NJ, Roeters JJ, Burgerdijk RC. Microleakage of Class II box-type composite restorations. Am J Dent 1998; 11: 160-4.

[21] Cardoso PE, Placido E, Francci CE, Perdigao J. Microleakage of Class $\mathrm{V}$ resin-based composite restorations using five simplified adhesive systems. Am J Dent 1999; 12: 291-4.

[22] Hakimeh S, Vaidyanathan J, Houpt MI, Vaidyanathan TK, Von Hagen S. Microleakage in class V restorations: dyract restorative system. J Prosthet Dent 2000; 83: 194-203.

[23] Pashley DH, Tay FR. Scanning electron microscopy of the structure of smear layers in human dentin. Arch Oral Biol 1988; 33: 265-70.

[24] VanMeerbeek B. Comparative SEM and TEM examination of the ultrastructure of the resin-dentin interdiffusion zone. J Dent Res 1993; 72(2): 495-501.

[25] Wieczkowski G, Yu XY, Davis EL. Microleakage in various dentin bonding agent/composite resin systems. Oper Dent 1992; 17(Suppl 5): 62-7.

[26] Nakabayashi N, Takarada K. Effect of HEMA on bonding to dentin. Dent Mater 1992; 8: 125-30.

[27] Vaidyanathan J, Vaidyanathan TK, Yadav P, Linaras CE. Collagen-Ligand interaction in dentinal adhesion: Computer visualization and analysis. Biomaterials 2001; 22: 2911-20.

[28] Chan KC, Swift EJ. Marginal seal of new generation bonding agents. J Prosthet Dent 1994; 74: 420-3.

[29] Ferrari M, Mannocci F, Vichi A, Davidson CL. Effect of two etching times on the sealing ability of Clearfil Liner Bond 2 in class V restorations. Am J Dent 1997; 10: 66-70.

[30] de Silva Telles PD, Aparecida M, Machado M, Nor JE. SEM study of a self-etching primer adhesive system used for dentin bonding in primary and permanent teeth. Pediatr Dent 2001; 23(4): 315-20.

[31] Miller MB, Ed. Reality 2003, Volume 27: Chapter on Dentin Adhesives, pp. 241-95, Reality Publishing Company, Houston, TX 2003.

[32] Kubo S, Yokota H, Sata Y, et al. Microleakage of self-etching primers after thermal and load cycling. Am J Dent 2001; 14: 163-9.

[33] Yoshida Y, Nagakane K, Fukuda R, et al. Comparative study on adhesive performance of adhesive monomers. J Dent Res 2004; 83: 454-8.

[34] Fabianelli A, Vichi A, Kugel G, Ferrari M. Influence of selfetching-priming bonding systems on sealing ability of class II restorations: leakage and SEM evaluation. Paper presented at the annual meeting of the International Association for Dental Research. Washington, DC 2000.

[35] Nakabayashi N, Pashley DH. Hybridization of dental hard tissues, Quintssence, Tokyo 1998.

[36] Tay FR, Pashley DH. Aggressiveness of contemporary self-etching systems. I: Depth of penetration beyond smear layers. Dent Mater 2001; 17: 296-308.

[37] Tat FR, Pashley DH. Have dentin adhesives become too hydrophilic? J Can Dent Assoc 2003; 69: 726-31.

[38] Christensen GJ. Preventing postoperative tooth sensitivity in class I, II and V restorations. J Am Dent Assoc 2002; 133: 229-31. 